\title{
Dynamic Analysis of Nonuniform Beams on Elastic Foundations
}

\author{
S. Abohadima*, and M.H. Taha ${ }^{2}$ \\ Dept. of Eng. Math. And Physics, Faculty of Engineering, Cairo University, Giza, Egypt
}

\begin{abstract}
An analytical solution for the free vibration of a nonuniform flexural beam resting on an elastic foundation is obtained. The characteristics of the beam are assumed variable over the beam length while the soil is considered of Winkler type. A power distribution model is used to simulate the variations in the beam geometry, beam material and soil stiffness over the beam length. The fourth order differential equation of beam vibration under appropriate boundary conditions is transformed to the Bessel equation by factorization. Mode shapes and damped natural frequencies of the beam are obtained for wide range of beam-foundation system characteristics. Numerical comparison demonstrates that the present model results for uniform case agree with those found in literature. The present model analytical solutions may be used to verify the accuracy of other numerical and approximate solutions.
\end{abstract}

Keywords: Damped vibration, tapered beam, differential equation, variable coefficients, series solution, mode shapes and natural frequencies.

\section{INTRODUCTION}

Many practical engineering applications as railroad tracks, highway pavement, buried pipelines and foundation beams are modeled as beams resting on elastic foundations. To investigate the dynamics of such applications, the vibration behavior of these models need to be accurately obtained. Few analytical solutions limited to special cases for vibrations of such models are found in literature. This is due to the difficult mathematical nature of the problem. Numerical methods such as finite element method [1-3], transfer matrix method [4], Rayleigh-Ritz method [5], differential quadrature element method (DQEM) [6-10], Galerkin procedure $[11,12]$ and perturbation techniques [13-15] are used to obtain the vibration behavior of different types of linear or nonlinear beams resting on linear or nonlinear foundations. Semi-analytical methods such as series solutions are suggested to obtain analytic expressions for frequencies and mode shapes of nonuniform beams resting on elastic foundation. Ruta P. [16] employed Chebychev series to obtain closed analytical formulae for the sought solution's coefficients. Calio I. and Elishakoff I. [17] solved the semi inverse problem to obtain the distribution of flexural rigidity and material density for a postulated vibration mode.

As an attempt to obtain analytical solution for such problem, Taha M.H. [18] studied the transient response of a finite prismatic beam resting on viscoelastic foundation under stochastic dynamic load using eigen function expansion. Also, Taha M.H. [19] obtained a closed form solution for damped free vibration of a non-uniform shear beam resting on an elastic foundation. Taha M.H. and Abohadima S. [20] have solved the fourth order differential equation with variable coefficient representing the vibrational behavior of a flexural beam and obtained solutions for a wide range of practical applications.

*Address correspondence to this author at the Dept. of Eng. Math. And Physics, Faculty of Engineering, Cairo University, Giza, Egypt; Tel: +20-2330 35072; Fax: +20-2-357 23486; E-mail: sabohadima@yahoo.com
In the present work, the model suggested in ref. [20] is extended to include the effect of foundation reaction on the vibration of nonuniform flexural beams. The foundation reaction is modeled as Winkler type. The beam mass intensity; damping intensity and flexural stiffness are assumed to vary as power functions along the beam length while the soil stiffness is assumed increasing linearly with the beam length. The fourth order differential equation with variable coefficients of the beam vibration is solved to obtain mode shapes and natural frequencies of the system. Charts of natural frequencies for wide range of non-uniform flexural beams resting on elastic foundation are conducted.

\section{ANALYSIS}

The vibration equation of a flexural nonuniform beam resting on an elastic foundation is given as:

$$
\begin{aligned}
& \frac{\partial^{2}}{\partial x^{2}}\left(k(x) \frac{\partial^{2} y}{\partial x^{2}}\right)+m(x) \frac{\partial^{2} y}{\partial t^{2}}+C(x) \frac{\partial y}{\partial t}+ \\
& k_{s}(x) y(x, t)=q(x, t)
\end{aligned}
$$

Where $k(x)$ is the flexural stiffness of the beam, $m(x)$ is the mass intensity, $C(x)$ is the material damping intensity, $k_{s}(x)$ is the foundation stiffness intensity, $q(x, t)$ is the vertical excitation acting on the beam, $y(x, t)$ is the vertical response of the beam, $\mathrm{x}$ is the coordinate axis along the beam and $t$ is time.

The Boundary conditions for a simply supported beam whose length is $L$ are given as:

$$
\begin{aligned}
& y(0, t)=0 \\
& y^{\prime \prime}(0, t)=0 \\
& y(L, t)=0 \\
& y^{\prime \prime}(L, t)=0
\end{aligned}
$$

where prime means derivative with respect to $x$. 


\section{PROBLEM SOLUTION}

To obtain the natural frequencies and mode shapes, one can assume:

$q(x, t)=0$

$\mathrm{y}(\mathrm{x}, \mathrm{t})=\mathrm{w}(\mathrm{x}) \mathrm{e}^{\mathrm{i} \Omega \mathrm{t}}$

where, $w(x)$ is the mode shape function and $\Omega$ is the complex damped natural frequency of the flexural beam. Substitution of Eqs. (3) and (4) into Eq. (1), yields:

$$
\frac{d^{2}}{d x^{2}}\left(k(x) \frac{d^{2} w}{d x^{2}}\right)-\left(m(x) \Omega^{2}-i C(x) \Omega-k_{s}(x)\right) w(x)=0
$$

Equation (5) is a fourth order differential equation with variable coefficients whose solution depends mainly on the distribution functions representing the beam characteristics and foundation stiffness. The flexural stiffness of the beam depends on the moment of inertia of the beam cross section while both the mass intensity and damping intensity depend on the area of the beam cross section. The foundation reaction depends on the foundation stiffness and the contact area between the beam and the foundations. Therefore, the system characteristics may be expressed as:

$$
\begin{aligned}
& k(x)=k_{o} \psi(x) \\
& m(x)=m_{o} \phi(x) \\
& C(x)=c_{o} \phi(x) \\
& k_{s}(x)=K_{s o} \phi(x)
\end{aligned}
$$

where $k_{o}$ is the flexural rigidity, $m_{o}$ is the mass intensity, $c_{o}$ is the damping intensity and $K_{s o}$ is the foundation stiffness intensity at $x=0$. Substituting Eqs. (6-9) into Eq. (5), one obtains:

$$
\frac{d^{2}}{d x^{2}}\left(\psi(x) w^{\prime \prime}\right)-\theta^{4} \phi(x) w(x)=0
$$

where:

$$
\theta^{4}=\frac{1}{k_{o}}\left(m_{o} \Omega^{2}-i c_{o} \Omega-K_{s o}\right)
$$

In the following analysis, a power function distribution is assumed to represent the case of a beam of rectangular cross section with variable width and depth; while the foundation stiffness increases along the beam length. However, the power distribution can represent the case of a circular pile with variable radius embedded in a soil whose stiffness increases linearly with depth.

The non-uniform characteristics of the beam-foundation system may be assumed as:

$\psi(x)=(1+\alpha x)^{n+2}$

$\phi(x)=(1+\alpha x)^{n}$

where the parameters $\alpha$ and $n$ are used to approximate the actual non-uniformity of the system. Substitution of Eqs. (12), (13) into Eq. (10), leads to:

$$
\begin{aligned}
(1+\alpha x)^{2} \frac{d^{4} w}{d x^{4}}+ & 2 \alpha(n+2)(1+\alpha x) \frac{d^{3} w}{d x^{3}} \\
+ & \frac{\alpha^{2}(n+2)(n+1)}{(1+\alpha x)} \frac{d^{2} w}{d x^{2}}-\theta^{4} w(x)=0
\end{aligned}
$$

Equation (14) may be rewritten in the operational form as:

$\left(\Delta+\theta^{2}\right)\left(\Delta-\theta^{2}\right) w(x)=0$

where the differential operator $\Delta$ is obtained as:

$$
\Delta=\alpha(n+1) \frac{d}{d x}+(1+\alpha x) \frac{d^{2}}{d x^{2}}
$$

The general solution of Eq. (16) is the sum of the solutions of the two Equations;

$$
\begin{aligned}
& (1+\alpha x) \frac{d^{2} w_{1}}{d x^{2}}+\alpha(n+1) \frac{d w_{1}}{d x}+\theta^{2} w_{1}(x)=0 \\
& (1+\alpha x) \frac{d^{2} w_{2}}{d x^{2}}+\alpha(n+1) \frac{d w_{2}}{d x}-\theta^{2} w_{2}(x)=0
\end{aligned}
$$

Introducing new variables $\xi(\mathrm{x})$ and $\mathrm{Z}_{\mathrm{i}}(\xi), \mathrm{i}=1,2$ as:

$$
\begin{aligned}
& \xi(x)=(1+\alpha x)^{\mu} \\
& Z_{i}(\xi)=\xi^{-v} w_{i}(x)
\end{aligned}
$$

Using the new variables (Eqs. 19 and 20), Eqs. (17) and (18) can be transformed to:

$$
\begin{aligned}
& \frac{d^{2} Z_{1}}{d \xi^{2}}+\frac{1}{\xi} \frac{d Z_{1}}{d \xi}+\left(\eta^{2}-\frac{v^{2}}{\xi^{2}}\right) Z_{1}(\xi)=0 \\
& \frac{d^{2} Z_{2}}{d \xi^{2}}+\frac{1}{\xi} \frac{d Z_{2}}{d \xi}-\left(\eta^{2}+\frac{v^{2}}{\xi^{2}}\right) Z_{2}(\xi)=0
\end{aligned}
$$

where the transformation parameters $\mu, v$ and $\eta$ are obtained as:

$\mu=\frac{1}{2}$

$v=-n$

$\eta=2 \theta / \alpha$

Equations (21) and (22) are the Bessel's and modified Bessel's differential equations of order $\mathrm{n}$ with parameter $\eta$ whose general solutions are given as [21]:

$$
\begin{aligned}
& w_{1}(x)=(1+\alpha x)^{-\frac{n}{2}}\left[B_{1} J_{n}(\eta \xi)+B_{2} Y_{n}(\eta \xi)\right] \\
& w_{2}(x)=(1+\alpha x)^{-\frac{n}{2}}\left[B_{3} I_{n}(\eta \xi)+B_{4} K_{n}(\eta \xi)\right]
\end{aligned}
$$

where, $J_{n}, Y_{n}$ are the Bessel functions of the first and second kind and $I_{n}, K_{n}$ are the modified Bessel functions of the first and second kind of order n. Using Eqs. (15), (26) and (27), the general solution of Eq. (14) may be written as: 
Table 1. Dimensionless Natural Frequencies of Uniform Beam Resting on Elastic Foundation $\left(\lambda=\mathbf{2 0}, \mathbf{k}_{\mathrm{s}}=1 \mathrm{E9} \mathbf{N} / \mathrm{m}^{3}, \rho=2500 \mathrm{~kg} / \mathrm{m}^{3}\right.$, $\mathrm{E}=2.1 \mathrm{e10}, \mathrm{b}=\mathbf{0 . 3}, \mathrm{h}=\mathbf{0 . 6}$ )

\begin{tabular}{|c|c|c|c|c|c|}
\hline $\mathbf{k}_{\mathrm{s}}$ & $\omega^{*}$ & $r=1$ & $r=2$ & $r=3$ & $r=4$ \\
\hline 0 & present & 3.0 & 12.7 & 27.6 & 48.6 \\
\hline 1E9 & present & 67.9 & 68.8 & 72.8 & 82.1 \\
\hline
\end{tabular}

$w(x)=(1+\alpha x)^{-\frac{n}{2}}\left[B_{1} J_{n}(\eta \xi)+B_{2} Y_{n}(\eta \xi)+B_{3} I_{n}(\eta \xi)+B_{4} K_{n}(\eta \xi)\right]$

The constants $B_{i}, i=1,2,3,4$ are obtained from the boundary conditions (Eq. 2) at the ends of the beam.

\section{MODE SHAPES AND NATURAL FREQENCIES}

Using the boundary conditions at the beam ends, the constants $B_{i} i=1,2,3,4$ can be obtained. Then, the frequency equation, the mode shapes and the natural circular frequencies of the beam-foundation system are obtained.

Applying the boundary conditions (Eq. 2), in the mode function (Eq. 28) leads to a linear system of algebraic homogeneous equations whose coefficients are Bessel's functions and its derivatives at $\mathrm{x}=0$ and $\mathrm{x}=\mathrm{L}$. This system may be expressed as:

$\mathbf{A} \mathbf{B}=0$

where $\mathbf{A}$ is the coefficients matrix and $\mathbf{B}$ is the constants vector. The condition of nontrivial solution of this system leads to the frequency equation as:

Det $\mathbf{A}=0$

The above equation is a nonlinear algebraic equation in $\eta$ $(\eta=2 \theta / \alpha)$ which may be solved using any appropriate iterative technique to obtain the roots $\eta_{\mathrm{r}}$, and then the natural frequencies of the system can be determined.

Substituting the obtained values of $\eta_{\mathrm{r}}$ in Eq. (29) and assuming any arbitrary value for the constant $B_{1}$ (say $B_{l}=1$ ), relative values of constants $B_{2}, B_{3}$ and $B_{4}$ can be obtained. Then, the r-mode shape can be obtained and drawn for any values of the system characteristics as shown in Fig. (1).

\subsection{Natural Frequencies}

Using Eqs. (11, 25), the r-mode complex damped frequency is obtained as:

$\Omega_{r}=\frac{i C_{o}}{2 m_{o}}+\sqrt{\frac{\alpha^{4} \eta_{r}^{4} k_{o}+16 K_{s o}}{16 m_{o}}-\frac{C_{o}^{2}}{4 m_{o}^{2}}}$

The first term of Eq. (31) represents the attenuation of the system vibration due to damping, while the second term represents the actual natural frequency of the r-mode. The $r$ mode damped natural frequency of the system may be expressed as:

$\omega_{d r}=\omega_{r} \sqrt{1-d^{2}}$ where:

$$
\begin{aligned}
& \omega_{r}=\sqrt{\frac{k_{o} \eta_{r}^{4} \alpha^{4}+16 K_{s o}}{16 m_{o}}} \\
& d=\frac{C_{o}}{C_{c}} \\
& C_{c}=2 m_{o} \omega_{r}
\end{aligned}
$$

where, $\omega_{\mathrm{r}}$ is the undamped natural frequency of $\mathrm{r}$-mode, $\mathrm{d}$ is the damping ratio and $\mathrm{C}_{\mathrm{c}}$ is the critical damping coefficient of the beam-foundation system.

\section{NUMERICAL RESULTS}

Since the obtained solution is expressed in a closed form, it does not need any additional corroboration [17]. However, for convenience, Table $\mathbf{1}$ indicates the results obtained from the present solution and those found in literature [22] for the case of a uniform beam resting on an elastic foundation.

The dimensionless frequency is defined as:

$\omega_{r}^{*}=\omega_{r} \sqrt{\frac{L^{4} \rho}{E h_{o}^{2}}}$

The mode shapes are shown in Fig. (1) when the beam depth, width and foundation stiffness increase linearly with beam length $(n=2, \alpha=0.2)$. It is clear that the amplitude of vibration decreases when the $\mathrm{x}$ increases, as the rigidity of the beam-foundation system increases. However the effect of non-uniformity is more obvious in higher modes.

The effect of non-uniformity coefficient $\alpha$ on the vibration amplitude is indicated in Fig. (2) for the first and second modes. As it is expected, as $\alpha$ increases the vibration amplitude decreases because the system becomes more rigid. Also the effect of $\alpha$ becomes stronger as $r$ increases.

Fig. (3) shows the effect of beam-foundation system nonuniformity on its dimensionless natural undamped frequencies. This is for beam-foundation system with slenderness ratio $\lambda=20$ and for two cases of foundation stiffness. The first for a beam without foundation $\left(\mathrm{k}_{\mathrm{so}}=0\right)$ and the second for a beam resting on a relatively rigid soil $\left(\mathrm{k}_{\mathrm{so}}=1 \mathrm{E} 9\right)$. As the foundation stiffness increase; the system becomes more rigid and the dimensionless frequencies increase. It is clear that the effect of foundation stiffness is more noticeable in lower modes which used in practical applications. 


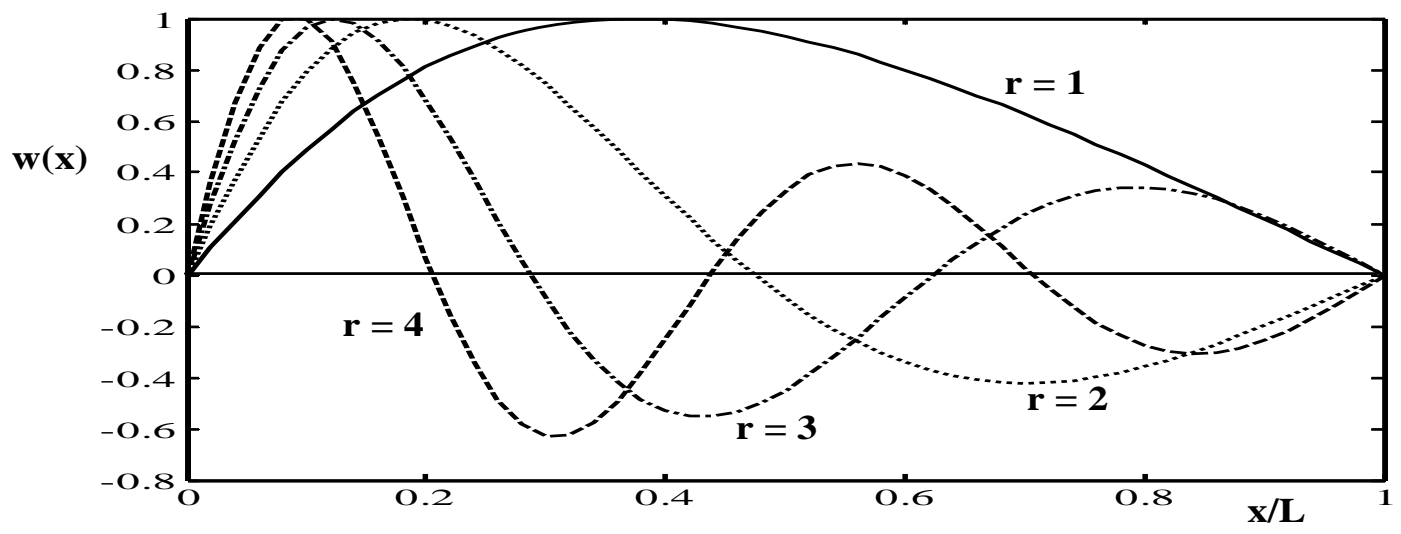

Fig. (1). Mode shapes for a nonuniform beam $(\alpha=0.2, \mathrm{n}=2, \lambda=20)$.

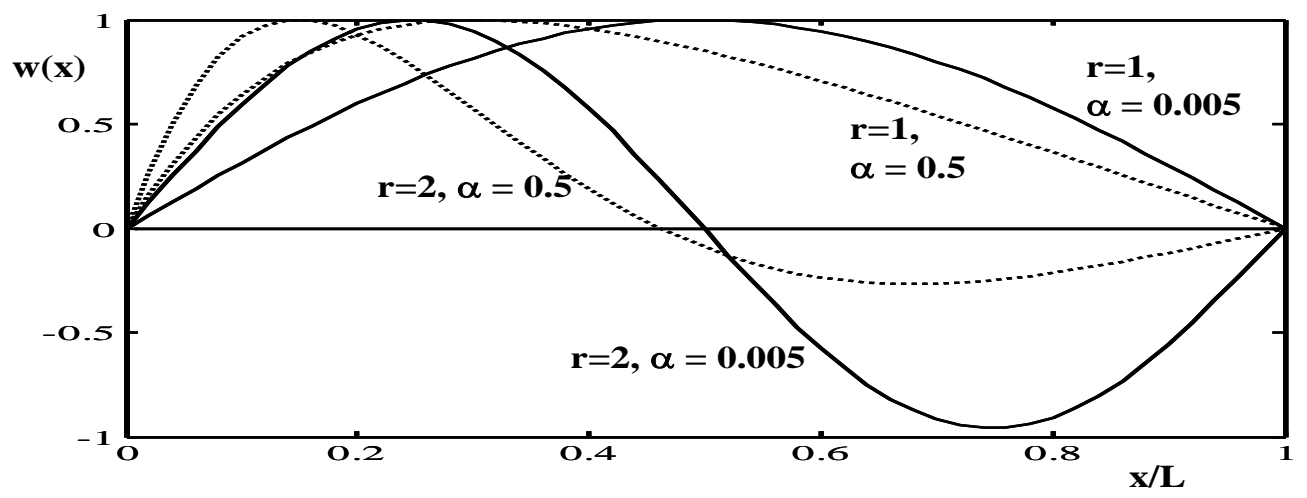

Fig. (2). Effect of nonuniformity factor $\alpha$ on the amplitude $(r=1,2 ; n=2 ; \lambda=20)$.

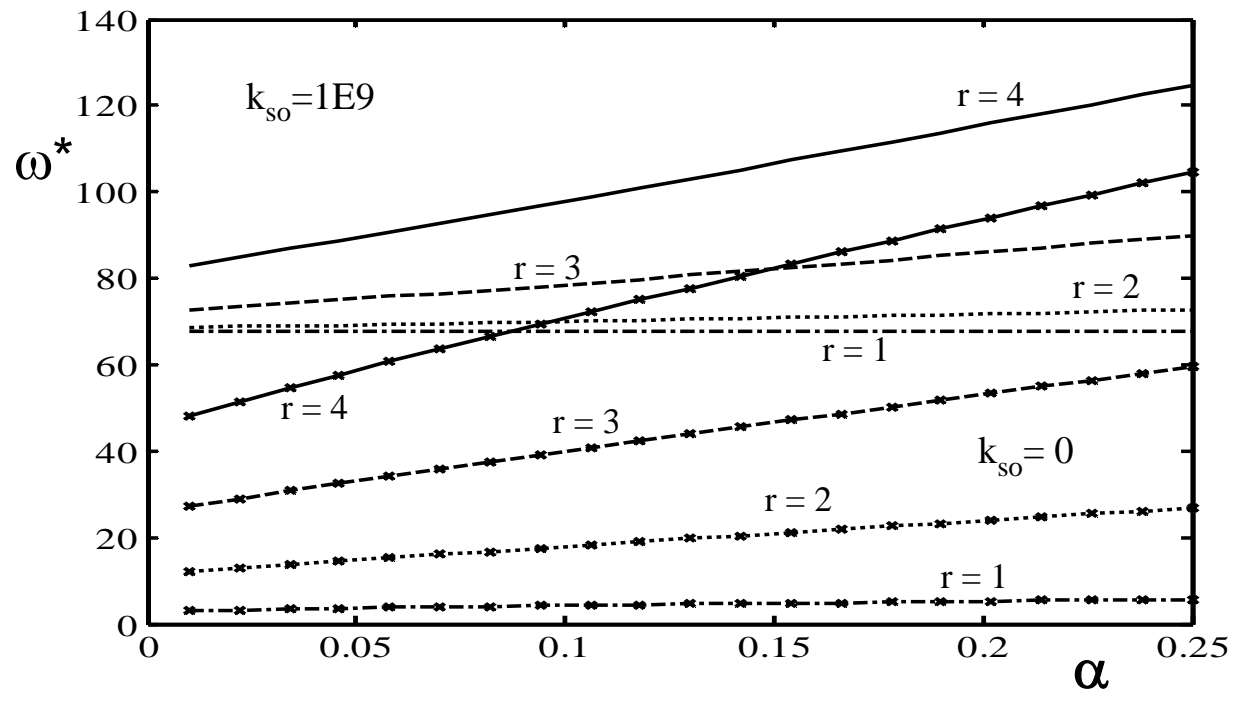

Fig. (3). Variation of the first four frequencies with $\alpha(n=2, \lambda=20)$.

Fig. (4) shows the effect of the slenderness ratio $\lambda$ on the dimensionless fundamental natural undamped frequency. This is for different values of non-uniformity coefficients $\alpha$ for two cases of soil as in Fig. (3). The graph may be considered as a design chart to calculate the fundamental natural frequency for beams with wide range of practical characteristics. However, the figure indicates that the influence of $\lambda$ is more noticeable for the case of beam with foundation. However, as $\lambda$ increases, the beam becomes more flexible.
Then, the existence of foundation increases the overall rigidity of the beam-foundation system.

\section{CONCLUSIONS}

Analytic expressions for the vibrational behavior of nonuniform viscoelastic flexural beam resting on an elastic foundation are derived. The characteristics of beamfoundation system are assumed to vary with the length as power functions. The dynamic equation of the beam- 


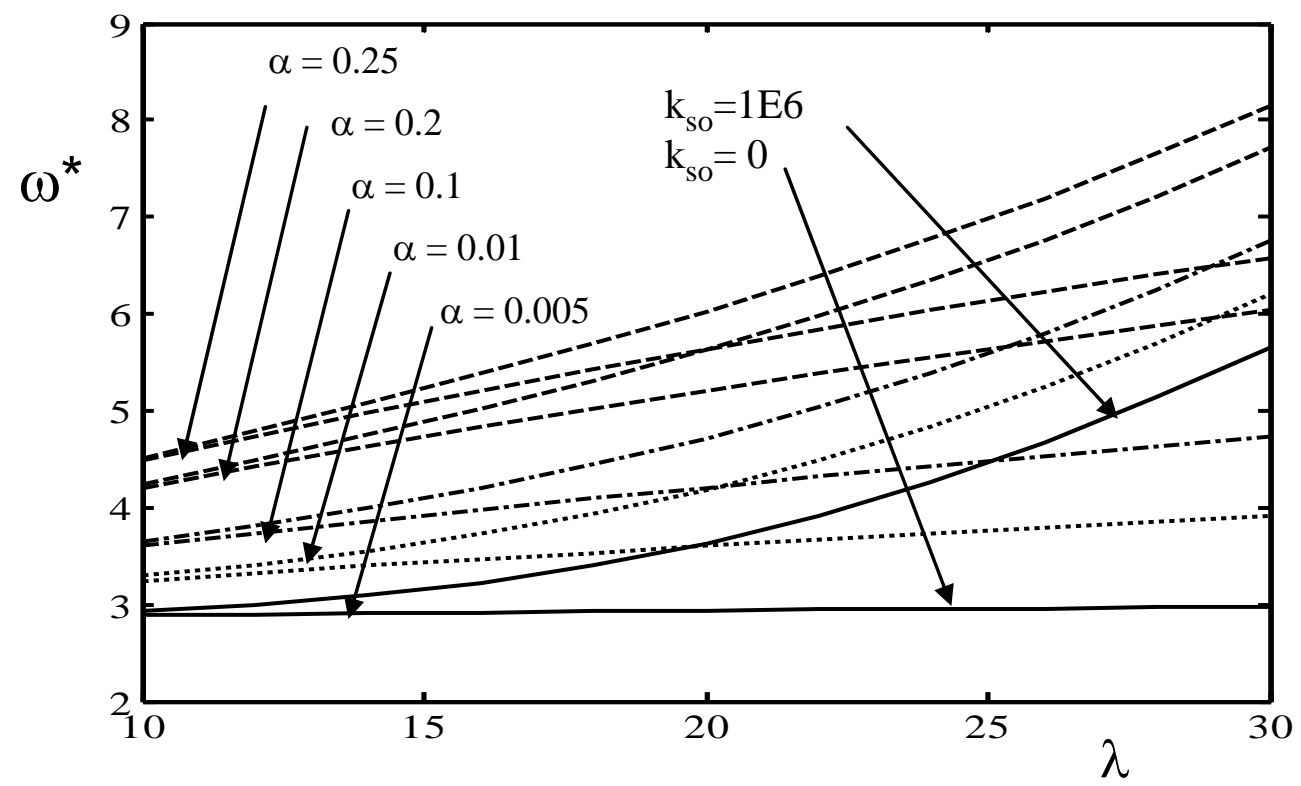

Fig. (4). Variation of fundamental natural frequency $(r=1)$ with slenderness ratio $\lambda(n=2)$.

foundation system is solved by introducing new variables to transform the dynamic equation to the Bessel differential equations. The suggested model is used to obtained solutions for mode shapes and natural frequencies for non-uniform beam-foundation system with linearly increasing depth, width and foundation stiffness $(n=2)$.

It is concluded that the effect of non-uniformity is more noticeable on frequencies of higher modes. The effect of foundation stiffness is more pronounced on frequencies of lower modes. Charts are presented to depict the variations in the dimensionless fundamental natural frequency for the non-uniform beam-foundation system. This is for beams with different variations in geometry and material characteristics which makes it useful for design purposes.

\section{REFERENCES}

[1] Lai YC, Ting BY, Lee WS, Becker WR. Dynamic response of beams on elastic foundation. J Struct Eng ASCE 1992; 118: 85358.

[2] Thambiratnam D, Zhuge Y. Free vibration analysis of beams on elastic foundations. Comput Struct 1996; 60 (6): 971-80.

[3] Ayvaz Y, Ozgan K. Application of modified Vlasov model to free vibration analysis of beams resting on elastic foundations. J Sound Vib 2002; 255(1): 111-27.

[4] Irie T, Yamada G, Takahashi I. Vibration and stability of a nonuniform Timoshenko beam subjected to a flower force. Sound Vib 1980; 70: 503-12.

[5] Gutierrez RH, Laura PAA, Rossi RE. Fundamental frequency of vibration of a Timoshenko beam of nonuniform thickness. J Sound Vib 1991; 145: 241-5.

[6] Chen CN. Vibration of prismatic beam on an elastic foundation by the differential quadrature element method. Comput Struct 2000; 77: 1-9.
[7] Chen CN. DQEM Vibration analysis of non-prismatic shear deformable beams resting on elastic foundations. J Sound Vib 2002; 255 (5): 989-99.

[8] Malekzadeh P, Karami G, Farid M. DQEM for free vibration analysis of Timoshenko beams on elastic foundations. Comput Mech 2003; 31: 219-28.

[9] Karami G, Malekzadeh P, Shahpari SAA. DQEM for free vibration of shear deformable nonuniform beams with general boundary conditions. Eng Struct 2003; 25: 1169-78.

[10] Chen WQ, Lu CF, Bain ZG. A mixed method for bending and free vibration of beams resting on Pasternak elastic foundations. Appl Math Model 2004; 28: 877-90.

[11] Pellicano F, Mastroddi F. Nonlinear dynamics of a beam on elastic foundation. Nonlinear Dyn 1988; 14: 335-55.

[12] Qaisi MI. Nonlinear normal modes of a continuous system. J Sound Vib 1998; 209 (4): 561-69.

[13] Nayfeh AH, Nayfeh SA. On nonlinear modes of continuous systems. Vib Acous ASME 1994; 116: 129-36.

[14] Maccari A. The asymptotic perturbation method fro nonlinear continuous systems. Nonlinear Dyn 1999; 19: 1-18.

[15] Coskun I, Engin H. Nonlinear vibrations of a beam on an elastic foundation. J Sound Vib 1999; 223 (3): 335-54.

[16] Ruta P. The vibration of a non-prismatic beam on an inertial elastic half-plane. J Sound Vib 2004; 275: 533-56.

[17] Calio I, Elishakoff I. Closed form trigonometric solutions for inhomogeneous beam-column on elastic foundation. Int J Struct Stab Dyn 2004; 4 (1): 139-46.

[18] Taha MH. Transient response of finite elastic beam on viscoelastic foundation under stochastic dynamic loads. Int J Differ Eqs Appl Sci 2002; 6/3: 283-97.

[19] Taha MH. Vibrations of nonuniform shear beams resting on elastic foundation. Eng Appl Sci 2004; 51(5): 843-55.

[20] Taha MH, Abohadima S. Mathematical model for vibrations of nonuniform flexural beams. Eng Mech 2008; 15 (1): 3-11.

[21] Tranter CJ. Bessel functions with some Physical applications. London: The English university press 1968.

[22] James ML, Smith GM, Wolford JC, Whaley PW. Vibration of mechanical and structural systems. New York: Harper Collins College Publishers 1994. 